(C) 2018 IEEE. Personal use of this material is permitted. Permission from IEEE must be obtained for all other uses, in any current or future media, including reprinting/republishing this material for advertising or promotional purposes, creating new collective works, for resale or redistribution to servers or lists, or reuse of any copyrighted component of this work in other works.

Kara, Peter A., Tamboli, Roopak R., Cserkaszky, Aron, Martini, Maria G., Barsi, Attila and Bokor, Laszlo (2019) The viewing conditions of light-field video for subjective quality assessment. In 2018 International Conference on 3D Immersion (IC3D) : proceedings. Piscataway : IEEE. ISSN: 2379-1780 ISBN: 9781538675908.

https://doi.org/10.1109/IC3D.2018.8657881 


\title{
THE VIEWING CONDITIONS OF LIGHT-FIELD VIDEO FOR SUBJECTIVE QUALITY ASSESSMENT
}

\author{
Peter A. Kara ${ }^{a b c}$, Roopak R. Tamboli ${ }^{\text {bd }}$, Aron Cserkaszky ${ }^{b}$, \\ Maria G. Martini ${ }^{a}$, Attila Barsi ${ }^{b}$, Laszlo Bokor ${ }^{C}$ \\ ${ }^{a}$ WMN Research Group, Kingston University, Kingston upon Thames, UK \\ Email: \{p.kara, m.martini\}@kingston.ac.uk \\ ${ }^{\text {b}}$ Holografika, Budapest, Hungary \\ Email: \{p.kara, r.tamboli, a.cserkaszky, a.barsi\}@ holografika.com \\ ${ }^{c}$ Budapest University of Technology and Economics, Budapest, Hungary \\ Email: $\{$ kara, bokorl $\} @$ hit.bme.hu \\ ${ }^{d}$ Indian Institute of Technology Hyderabad, India \\ Email: ee13p0008@iith.ac.in
}

\begin{abstract}
At the time of this paper, research on the quality of lightfield visualization is continuously gaining momentum, as the excellence in visual performance shall be one of the key enablers of the emergence and long-term presence of this technology on the consumer market. Experiments on perceived quality began in parallel with the appearance of commercially available light-field displays. As the techniques of subjective quality assessment on these displays are not standardized yet, certain parameters may greatly vary, such as viewing conditions. Although most of the current research efforts in this area address forms of static visualization, light-field video is also an essential target of quality evaluation. In this paper, we introduce the results of an experiment investigating the viewing conditions of light-field video quality assessment. The tests directly compare the perceived horizontal motion parallax of static viewing positions with motion patterns. The visual quality of the test conditions was degraded by the reduction of content spatial and angular resolution, as they both affect the smoothness of the horizontal motion parallax.
\end{abstract}

Index Terms - Light-field video, perceived quality, angular resolution, parallax effect.

\section{INTRODUCTION}

Although the ultimate visual experience of glasses-free 3D display systems demands the parallax effect to apply to both

The work in this paper was funded from the European Union's Horizon 2020 research and innovation program under the Marie Sklodowska-Curie grant agreements No 643072, Network QoE-Net and No 676401, European Training Network on Full Parallax Imaging, and it was also supported by the Laboratory of Multimedia Networks and Services (MEDIANETS). horizontal and vertical directions simultaneously — such systems are commonly known as full-parallax (FP) displays the horizontal-parallax-only (HPO) light-field displays of the present day can already achieve a high level of viewer satisfaction regarding this aspect. First of all, our eyes are horizontally separated, thus the eyes meet different light rays, describing the visualized content from slightly different angles. It is crucial to add here that the perception of the parallax effect does not require two separate eyes. While observing a given portion of the real world in a standing or sitting position, a living, breathing human being always has a measurable extent of natural head movement, no matter how much one concentrates on being still. Evidently, if the head moves, the eyes change their positions as well, compared to the target of observation. However, even if we assume that the head can be perfectly positioned into a fixed place, and only a single eye can collect visual information, the parallax effect will still be experienced. This is due to the movement of the eye. The only scenario in which the parallax effect is perceptually absent is when the eye is limited not only to a given position, but also to a fixed orientation.

Prior to the appearance of real light-field displays in the industry and in research, scientific efforts in the area have already addressed the topic of the horizontal parallax effect in practice [1] [2]. Ono et al. [3] proposed a display system in which the 3D-nature of the perception of visualization results from the synchronization between the movement and the rotation of the visual stimulus on a $2 \mathrm{D}$ screen and the movement and the rotation of the head of the observer. The implementation at hand was limited with regards to the speed of movement and rotation, as unsupported motion levels could hinder the 3D experience through the loss of synchroniza- 
tion, which was maintained via the help of a slowly-moving marker on the screen that the observer had to focus on and follow. Carballeira et al. [4] presented a novel model to capture the subjective perception of such 3D content, called the MultiView Perceptual Disparity Model (MVPDM), which takes into consideration the comfortable levels of the aforementioned movement and rotation speed and thus the smoothness in viewpoint transition. In this experiment, the parallax effect was simulated by view-sweeping on a stereoscopic display, therefore special 3D glasses had to be worn in order to perceive the content. Again, these works only deal with the horizontal component of the parallax effect; they are HPO solutions and do not take into consideration the vertical dimension. Wang et al. [5] combined the conventional integral imaging and an Augmented Reality (AR) technique in order to propose a $3 \mathrm{D}$ system that has the potential to be applied to a head-mounted display due to its small physical size. The lenslet display described in the work can be observed without any viewing equipment in a FP manner - both horizontal and vertical parallax is supported - however, both dimensions are significantly limited in the field of view (FOV).

Some say that people take certain things for granted. Although this is usually a topic of social philosophy — targeting family, friends, health and many other essential pieces of the puzzle of life - one thing that is definitely taken for granted is the smoothness of the parallax effect. As we observe the real world, the parallax effect is always as continuous and smooth as it can be, and nothing can really disturb it. Unfortunately, this does not necessarily apply to what display technology can imitate today. The parallax effect of light-field visualization is only virtually continuous, and its smoothness depends on the angular resolution of the visualized content and the display system itself. Failure to comply with the requirements set by the visuals of real life and its perception via the human visual system (HVS) can result in a severely degraded user experience, regardless of the use case scenario of the technology. Based on how insufficient the density of the displayed visual information is, a series of visual phenomena can degrade visualization, such as the crosstalk effect (when adjacent source views interfere with each other) or discrete view jumps (when there is a perceivable sudden shift between source views, without proper transition). The primary issue is that even a small extent of degradation can be capable of breaking the 3D immersion.

If the source views are captured by regular pinhole or virtual cameras - instead of lenslet cameras — then the HPO content is basically a series of 2D camera images, describing the scene from horizontally different positions. In lightfield visualization, the density of these images is the content angular resolution; the ratio of the number of views and the size of the valid FOV they are displayed in. In case of multi-view displays, there are multiple separate small FOVs — called "sweet spots" - and the content can be observed from the same viewing angle interval, regardless of the loca- tion of the sweet spot the observer is located in. The number of sweet spots provided by the display system determines the total number of simultaneous observers. Between the sweet spots, the observers can only perceive heavy crosstalk [6]. Light-field displays assign the entire FOV to a single, continuous visualization, therefore the content can be viewed from any angle within the FOV and perception shall change accordingly. Furthermore, there is no strict constraint regarding the number of simultaneous observers; as long as all the observers are located within the valid FOV.

The smoothness of the virtually-continuous horizontal parallax is also affected by content spatial resolution, which is the image resolution of the aforementioned $2 \mathrm{D}$ views. Insufficient spatial resolution results in blur that is not uniform across the screen of the display, as light rays hit irregular positions. However, if content angular resolution is low as well and thus the parallax effect is disturbed, such blur can lessen the impact of the degradation. Even though this compensation effect is not decisively significant, it is indeed measurable [7], and therefore should be taken into consideration.

Of course research on light-field Quality of Experience (QoE) is not limited to the investigation of the perceived parallax effect, as there are many components of subjective quality that are to be studied. However, at the time of writing this paper, the viewing conditions of subjective quality assessment are not standardized, yet their role in research is rather apparent. The parallax effect of light-field displays is often referred to as horizontal motion parallax, as the sideways motion of the observer within the FOV can enable fundamentally different viewing angles of the same scene.

In this paper, we review the state-of-the-art procedures in research on the subjective quality assessment of light-field visualization, and present our novel experiment regarding the viewing conditions of light-field video. The paper categorizes research by visual stimulus type, as for example the assessment of a static 3D model may come with different requirements than a complex scene with notable motion vectors. Although the visualization of light-field content can be performed using apparatus (or apparatuses) of different display technologies, the paper only considers research using actual light-field displays. Furthermore, near-eye technology where the screen of the display is positioned only mere centimeters from the eyes of the human observer — such as the works of Lanman et al. [8] and Hansen et al. [9], are not covered either.

The remainder of this paper is structured as follows: the parameters of the related research using static scenes are introduced in Section 2. Section 3 covers interactive test scenarios, where actions of the user(s) impact the visualized content. Considerations regarding the quality assessment of light-field video are detailed in Section 4. This section also presents the analysis of our subjective test findings on the topic. The paper is concluded in Section 5. 


\section{STATIC SCENE VISUALIZATION}

Static scenes are the most common content types of lightfield visualization at the time of this paper. Static content can either be a single object or multiple objects that together form a scene. Such contents are presented to the observer at a given orientation and level of zoom, that can differ between test stimuli, according to the investigated test conditions. The given stimulus does not vary over time, and the observer has no direct interaction with the visual presentation of the content.

As the content is still, recording it for light-field visualization offers the ease of single-camera solutions. If the physical dimensions and weight of the object or scene are fairly limited, the turntable solution becomes viable. It means that the single pinhole camera remains in a fixed position during capture, and the targeted content is placed on a turntable, which rotates around its axis. A recent example for such lightfield content is the high-angular-resolution turntable dataset of Tamboli et al. [10].

In the earliest published research efforts addressing subjective visual quality, viewing conditions were not necessarily taken into consideration, and thus in certain papers they were not properly reported. The generally accepted procedure enabled arbitrary observer movement within the valid FOV, so that the content can be perceived from several different viewing angles and distances.

Static content (just like any other content) can indeed look fundamentally different from various angles - of course if and only if the content and display FOV support such change. An example of this is given in Figure 1, where 5 cards are displayed on the HoloVizio 80WLT, which has a valid FOV up to 180 degrees. In our earlier research on FOV size [11], observers could freely move inside a semicircle around the display, with a radius of 5 meters. It was crucial to enable such freedom of movement, as the stimuli differed in FOV, and the observers themselves had to discover the given FOV size and rate accordingly.

It is important to note that the type of the display itself can create constraints for viewing conditions, by redefining the valid FOV. In practice we categorize light-field displays as either back-projection or front-projection systems. Backprojection displays are similar to all conventional 2D televisions, in the sense that optical engines are located on one side of the screen, and the observer perceives the screen from the other side. Such displays can even be perceived from a distance in the order of tens of centimeters. This does not apply to front-projection systems, where the optical engines are on the same side of the screen as the observer. In this case, the valid FOV is affected (reduced) by the fact that if the observer comes too close to the screen, occlusion may occur with the rays emitted by the optical engine, resulting in the loss of visual information on the screen. For instance, in the previously mentioned subjective tests using the HoloVizio 80WLT

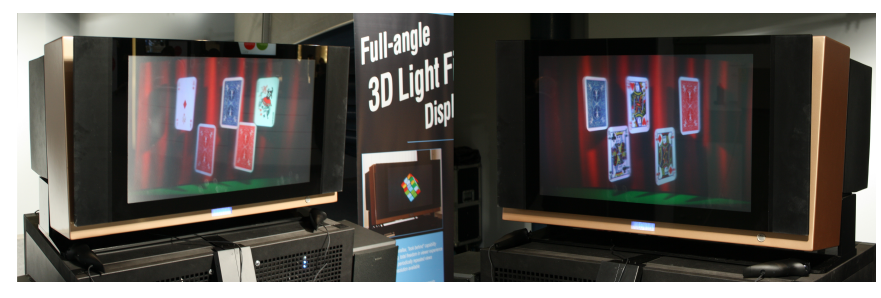

Fig. 1: Static content visualized on the HoloVizio 80WLT.

(back-projection display), observers could go as close to the display as they wished, while in research using the HoloVizio C80 (front-projection display), generally observers could not approach the screen closer than the position of the optical engine array.

Tamboli et al. [12] used $1.7 \mathrm{H}$ as viewing distance (1.7 times the height of the screen of the light-field display) with fixed observer positions in 5 viewing angles (standing observers). The distance of these positions was measured from the center point of the screen, thus they were placed along an arc. During the subjective tests, no separation screens were used, which means that at every given point in time, there was a stimulus displayed on the screen of the light-field display. The relevance of this information is that even though the stimuli were only assessed from the fixed positions, observers could see the visualized content in its given quality while moving between these positions. Such visual information can influence quality ratings, and should be taken into consideration.

In our prior research on the angular resolution of static visual stimuli [13], observers were $2.5 \mathrm{H}$ away from the screen, with a sideways movement of 2 meters. In a different work using the same display [14], 6 positions (seats) were selected: 3 of them at $2.5 \mathrm{H}$ and another 3 at $3 \mathrm{H}$, with a sideways 1 meter separation between them. The results of the latter work have shown more tolerance towards visual disturbances.

Note that there are two main approaches for position selection. One is arc (e.g., [12]), where distance is measured from the center of the screen, and one is linear (e.g., [14]), where distance is measured from the plane of the screen. In many cases, the practical difference in viewing distance between these approaches is nearly negligible, due to the small curvature of the arc.

In our work on spatial resolution, a rectangular area of arbitrary movement was set, beginning at $2.5 \mathrm{H}$ from the display. The initial viewing distance for each stimulus was always $2.5 \mathrm{H}$, and then viewing distance and angle could be changed for more accurate observation. The test design for the subjective assessment originates from the preliminary tests, during which multiple observers reported the need for increased viewing distances, especially for highly blurred content.

Apart from a very limited number of examples, for all works involving the HoloVizio C80 front-projection light-field display, the viewing distance of $2.5 \mathrm{H}$ was commonly used. 
This applies to the experiments of Cserkaszky et al. [15] [16] and Darukumalli et al. [17] as well. As a counter example, Kovacs et al. [18] used 2.7H. Greater viewing distances did appear in literature, but smaller ones not even once, due to the front-projection nature of the system.

\section{INTERACTIVE VISUALIZATION}

Interactive visualization refers to subjective tests where the observer directly engages with the content in a given way, and the interaction may result in a change of the shown content. A recent example for this is the work of Tamboli et al. [19], investigating 3D orientation preference. Observers used a gamepad to rotate the given object, but other interactive features - such as zooming or panning - were not enabled. The tests were carried out on the HoloVizio C80, using the default $2.5 \mathrm{H}$ viewing distance. However, sideways movement was essential to the study, as the involvement of the parallax effect via glasses-free 3D visualization was one of the primary contributions compared to 2D and stereoscopic $3 \mathrm{D}$ works in the literature. Images of the test procedure are shown in Figure 2.

The work of Adhikarla et al. [20] [21] relied on hand gestures, tracked by Leap Motion. Interactivity included panning, rotating and zooming, and also surface touching on the screen of a small-scale light-field display. Although viewing conditions are not directly specified, the task of surface interaction implies the distance to be approximately an arm's reach. Beyond user experience, the research addressed task completion time and cognitive workload as well.

Light-field gaming is definitely a promising potential of the technology [22]. In such use cases, viewing conditions are not only affected by the type of the display system, but the control system as well. For example, let us take a real-time strategy game that is controlled via a keyboard and mouse combo. With such controllers, the user is most likely to be bound to a fixed position, with a given viewing distance and viewing angle (center view expected). A photo of such gaming application is shown in Figure 3.

However, certain games with special controllers and motion tracking require user movement. This means that research on such games may be rather challenging to cope with on the plane of viewing conditions, as different user movements - and thus the corresponding changes in viewing distance and angle - are essential parts of the gameplay itself.

Interactive light-field visualization also includes the use case of navigation and exploration. An example of an aerial city view is given in Figure 4 . The previously mentioned consideration applies here as well. In the shown example, a mouse was used to navigate the map, implying a fixed position. However, this can also be done with the help of a gamepad, enabling higher mobility, or even hand gestures or general motion tracking.

Real-time light-field 3D telepresence also belongs to the

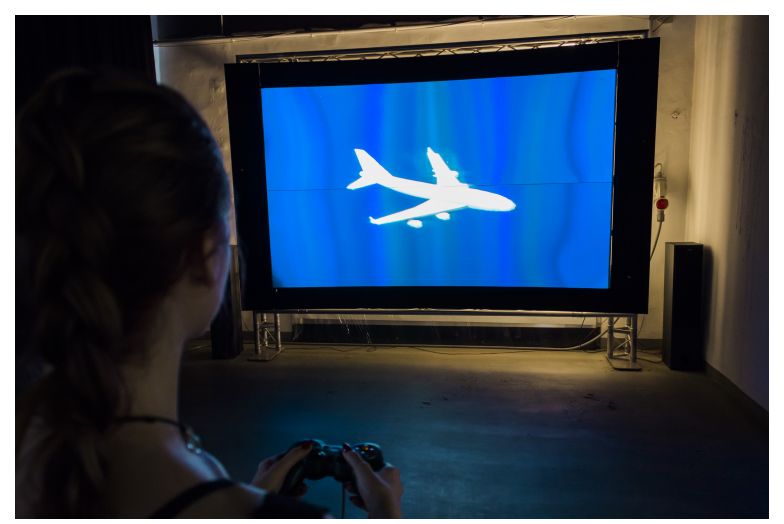

Fig. 2: Interactive 3D orientation preference selection [19] on the HoloVizio C80.

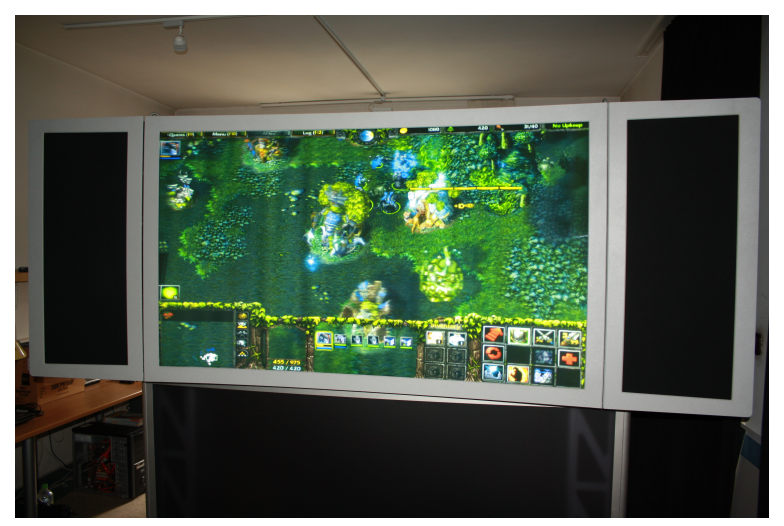

Fig. 3: Warcraft III (@)Blizzard Entertainment) gameplay on the HoloVizio HV640.

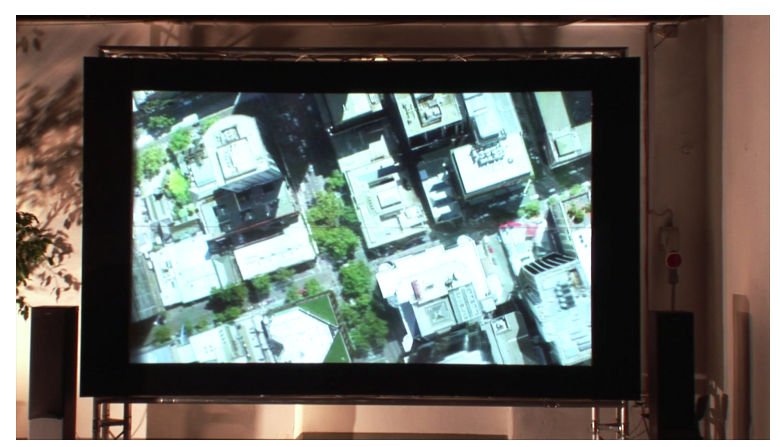

Fig. 4: Visualization of myVR on HoloVizio C80, showing an aerial view of London.

category of interactive use cases. In case of a one-way system - such as the work of Cserkaszky et al. [23] — viewing conditions are less constrained than in a symmetric system. A one-way asymmetric system captures the live light-field video feed of one individual, and shows it in real time to another. In this case, the observer on the receiver side only needs to stay within the valid FOV. If the system is a two-way symmetric 
one, then both users are captured and are shown to each other in real time. This means that the camera system has its own position constraint on the user. Of course in an ideal case, the capture and display components are perfectly aligned, therefore any given position within the valid FOV is more than sufficient for the capture system and vice versa.

Video watching is conventionally perceived as a passive use case, where the observer does not interact with the visualization of the content. However, interactive options - such as content rotation or zooming - are possible as well. In the scope of our research, we exclusively investigated the classic passive use case, results of which are presented in Section 4.

\section{LIGHT-FIELD VIDEO}

Light-field video is now emerging within the area of research, although it is still heavily outranked by the massive weight of static scene visualization. From the related work, the research of Adhikarla et al. [24] is quite notable, as the authors proposed a live capture system. Dricot et al. [25] investigated the feasibility of a light-field video service. In our earlier works on light-field video, we evaluated the concept of dynamic adaptive streaming [26] and particularly addressed video quality [27]. These works used the regular $2.5 \mathrm{H}$ viewing distance in default centered position, accompanied by a meter movement space in both sideways directions.

Dricot et al. [25] used 3.3H with 6 fixed positions (seats), and also moving observers with a sideways distance of 2 meters. As the authors collected ratings using a Double Stimulus Impairment Scale (DSIS) [28], it was possible to subjectively assess whether a given degradation was perceivable or not. Results indicate that quality disturbances were more observable during the specified sideways movement compared to fixed-position evaluation.

In our research to address viewing conditions, we directly compared fixed viewing positions with observer movement. As the HoloVizio C80 was used, one position was the default $2.5 \mathrm{H}$ center view, and two other positions were its 1 -meter sideways shifts (left and right).

For such systems, viewing the screen of the display from a significantly greater distance can result in the loss of the $3 \mathrm{D}$ experience. As the formulation of this statement is rather vague, let us convert this into specific facts and actual numbers. In case we consider an observer with two eyes, we can say that the $3 \mathrm{D}$ experience requires that the two eyes can be addressed with two separate light rays. Let us denote the display's angular resolution by $A R$, the average distance between the eyes of the observer as $D_{E}$, and the viewing distance at which the $3 \mathrm{D}$ experience is still supported as $D_{V}$. The rule of thumb in this case is

$$
D_{V}=\frac{D_{E}}{\tan (A R)}
$$

If we take $D_{E}$ as $6.5 \mathrm{~cm}$ and $A R$ as 0.5 degree, then $D_{V}$ is $745 \mathrm{~cm}$. However, (1) is calculated for a perfectly still human observer, which does not exist in practice. The general approach in industry is that still (i.e., not moving) observers can experience $3 \mathrm{D}$ visuals at $2 \times D_{V}$, and moving observer at $3 \times D_{V}$.

The experimental configuration means that a 25-degree portion was used of the total 45-degree valid FOV. Let us denote the left position as $A$, the center one as $B$ and the right one as $C$. The two linear motion patterns defined were $A C A$ and $C A C$, where $A C A$ means that during the lightfield video stimulus, the observer moved from $A$ to $C$, and then back to $A$. With normal-paced walking, such sideways movement was measured to be between 8 and 12 seconds, depending on the test participant in the preliminary tests. Therefore, the video content had to be around this interval.

We considered using clips from Blender's well-known Big Buck Bunny, which has already been accommodated to lightfield visualization by Kovacs et al. [29]. In the end, we decided to use the 5 different clips involved in our prior works on light-field video for multiple reasons. First of all, we found their durations to be adequate for the purpose of this experiment, as they fit into a range of 7 to 14 seconds. Second, these clips were created for and by Holografika, with emphasis on depth budget and motion. Lastly, they performed well in our prior researches, and using these videos make comparison possible with earlier findings.

A frame of each source stimulus - captured by a pinhole camera - is shown in Figure 5. The first two (Red and Yellow) were provided by Freelusion ${ }^{1}$ with the aim of using the depth budget of the light-field display to a great extent. The following two (Ivy and Tesco) were created by Post Edison ${ }^{2}$, targeting subtle and intense motions, respectively. The final source stimulus (Gears) was a looping animation, rendered by Holografika.

At first, the reference stimulus was shown for each content, with high angular ( 2 source views per degree) and spatial resolution (varied per content [27]). The two degraded test conditions were one with reduced angular resolution (1 source views per degree), and one with reduced angular and spatial resolution, denoted as LA-HS and LA-LS in the remainder of the paper, respectively. Note that according to (1), $2 \times D_{V}$ is $7.45 \mathrm{~m}$ if $A R$ is reduced to 1 degree, thus the $4.6 \mathrm{~m}$ corresponding to $2.5 \mathrm{H}$ distance was deemed suitable for $3 \mathrm{D}$ experience.

The HoloVizio C80 supports content angular resolution up to 0.5 degree, therefore no down-sampling was necessary during the conversion of the camera images, as the test conditions did not exceed this capability of the display. Downsampled content can achieve a different perceived quality than content captured or rendered to match the angular resolution of the display [13], but at the time of this paper, the literature

\footnotetext{
${ }^{1}$ Freelusion Video Mapping + Dance Company (http://freelusion.com/)

${ }^{2}$ Post Edison Computer Graphics (http://www.postedison.hu/)
} 

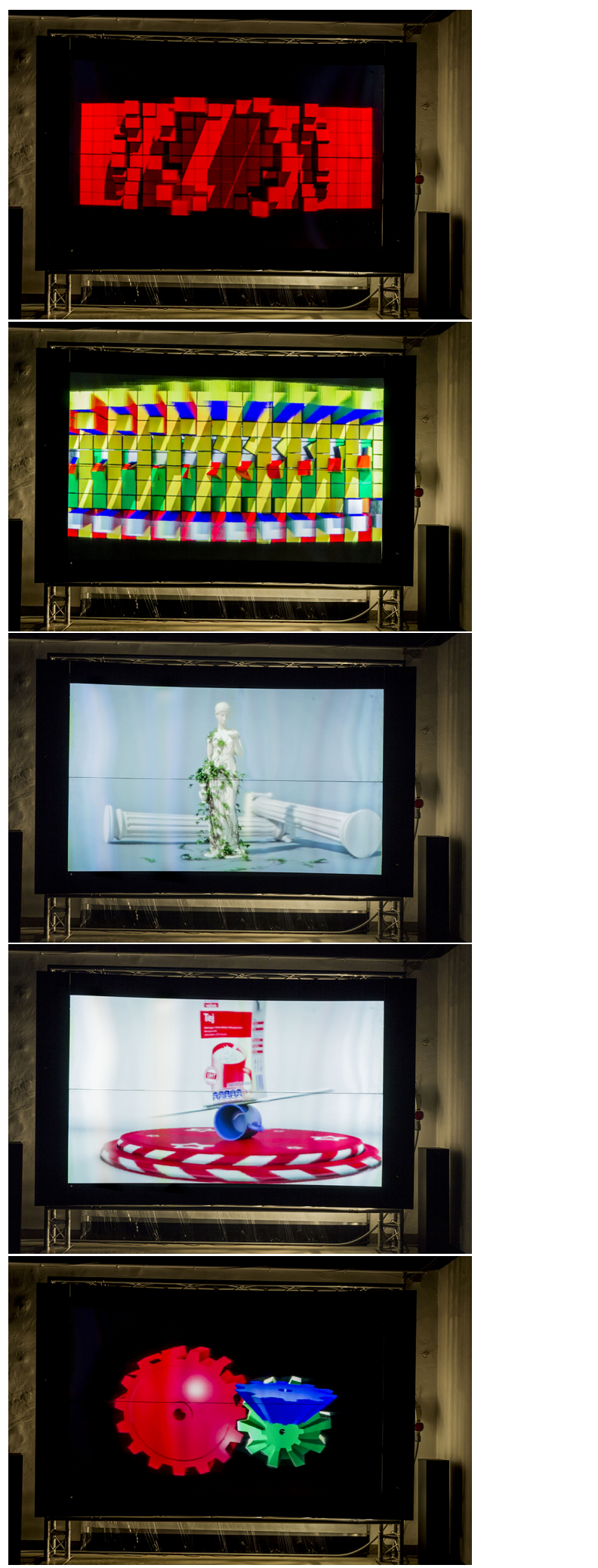

Fig. 5: Source video contents (Red, Yellow, Ivy, Tesco and Gears) visualized on the HoloVizio C80. lacks experiments addressing this matter. In the future, we do expect to encounter significantly more powerful display systems and higher-quality content as well. Although the topic of viewing conditions in general will remain a critical focus in research methodology, the special considerations regarding the smoothness of the parallax effect that are the scope of this paper will not necessarily apply if any given extent of disturbance can be avoided. However, instead, a new frontier of challenges (and the corresponding research questions) in perceived quality shall emerge as soon as FP displays become common in QoE experiments.

The series of subjective tests was carried out in a laboratory environment, isolated from external distractions. The lighting condition of the room was approximately 20 lux and the brightness of the screen was $1500 \mathrm{~cd} / \mathrm{m}^{2}$. Observers were selected from a pool of 18 people; a mix of experts and naïve test participants, from an age range between 20 and 42 .

The first test compared the $A C A$ and $C A C$ motion patterns. The research aim here was to check whether we need to compare both these patterns with the 3 fixed position or not. A binary scale was used to assess the perceived difference in the smoothness of the parallax effect. Results indicate that not a single observer was able to differentiate these patterns with regards to parallax smoothness, therefore we only used one in the exhaustive paired comparison test, whichever the observer preferred.

As there were 5 contents, 4 viewing conditions ( 3 fixed positions and 1 motion pattern) resulting in 3 direct comparisons, and 2 test conditions, an observer completed 30 paired comparison, using the 5-point Degradation Category Rating (DCR) scale [30]. Observers also provided extensive verbal feedback regarding what they perceived, assisting the understanding of the results.

The results of the subjective tests are introduced in Figure 6, in the forms of obtained scoring distribution and mean scores. First of all, only $36.92 \%$ of the scores were 5 (no perceivable difference in the smoothness of the horizontal parallax); $63.08 \%$ of the scores indicate a perceivable difference induced by observer motion. In fact, the number of 4 ratings (perceivable difference that does not annoy the observer) alone surpassed the number of 5 ratings. 3 ratings (slight annoyance) and 2 ratings (considerable annoyance) were at $21.8 \%$ and $4.1 \%$, respectively, and not a single observer used the bottom of the scale (high level of annoyance).

As for the two test conditions, the obtained results show a statistically significant difference. The 0.95 confidence intervals do not overlap, and $p<0.01$ applies to post-hoc Tukey HSD, Bonferroni and Holm tests. Therefore, the horizontal parallax was deemed to be smoother when spatial resolution was reduced as well (LA-LS). This is quite notably reflected in the scoring distribution as well. Roughly twice as many ratings registered annoyance for LA-HS than LA-LS, and the two test conditions also reached more than a $10 \%$ difference for 5 ratings. 

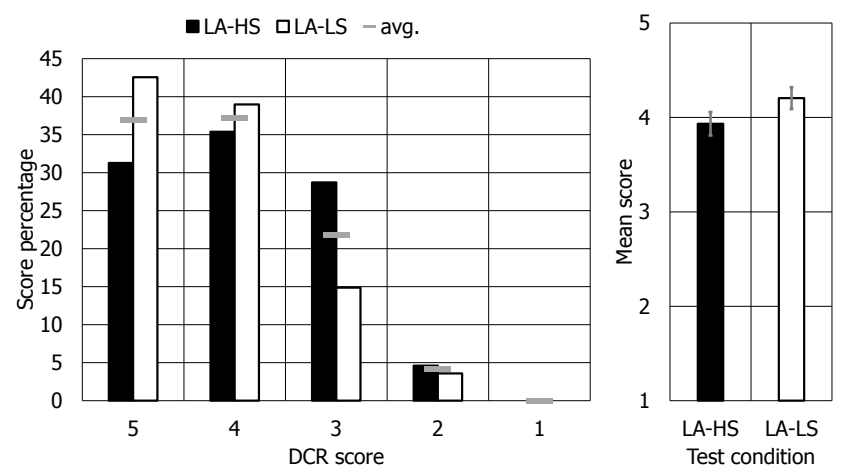

Fig. 6: Scoring distribution (left) for test conditions with low angular resolution and high spatial resolution (LA-HS), with low angular and low spatial resolution (LA-LS), and for all scores (avg.). Mean scores (right) for LA-HS and LA-LS.

Regarding the viewing positions ( $A, B$ and $C)$, no statistical difference was found. With 5 contents and 2 test conditions, there were $10 A B C$ triplets per observer. Nearly half, $47.7 \%$ of these triplets were without variations (all 3 scores were the same), $40 \%$ had a single different score and $12.3 \%$ had 3 different scores. However, due to the great variation in the scoring dissimilarities, there was no dominant pattern and the averages fit into an interval of 0.05 .

Finally, the findings for the selected source content are similar to viewing positions. Although there were indeed certain differences for content scores per observer, there was no obvious perceptual preference. For example, some did not perceive any degradation for Red and Yellow, while others not only perceived disturbances in the smoothness, but also rated them to be slightly annoying.

\section{CONCLUSION}

In this paper, we presented an overview of viewing conditions separately for static, interactive and video content for glassesfree 3D quality assessments, and introduced our own research on light-field video. The obtained results indicate a better tolerance towards low angular resolution for static, still human observers, compared to moving ones. The findings also show the impact of spatial resolution on the perceived smoothness of the horizontal parallax. This is in alignment with our results achieved from earlier experiments: in many cases, observers perceived that having both angular and spatial resolution reduced enabled a smoother parallax compared to the test condition when only angular resolution was reduced. As it has been stated earlier in the paper, this is due to the additional blur introduced by low spatial resolution. In the scope of this research, overall QoE was not addressed, thus only the beneficial effect of low spatial resolution was considered.

Our future research efforts in the topic of viewing conditions aim at investigating the greatest supported viewing dis- tances for different display systems and source content resolutions. Such scientific knowledge on perceived quality is vital for the design of future large-scale systems that simultaneously accommodate an exceptionally high number of observers (order of a hundred) [31]. We shall also investigate the 3D disparity of such visualization, using stereo cameras and objective metrics. Lighting conditions are to be addressed as well, since inadequately set environmental illumination may mask certain visual phenomena or alter general QoE. Finally, we intend to use real-world camera-captured content as well in future researches, particularly with complex scenes.

\section{REFERENCES}

[1] L. Onural, 3D Video Technologies: An Overview of Research Trends. SPIE, 2010.

[2] N. S. Holliman, N. A. Dodgson, G. E. Favalora, and L. Pockett, "Three-Dimensional Displays: A Review and Applications Analysis," IEEE Transactions on Broadcasting, 2011.

[3] H. Ono and S. Yano, "A 3-D display system using motion parallax," in Three-dimensional Imaging, Visualization, and Display, 2009.

[4] P. Carballeira, J. Gutirrez, F. Morn, J. Cabrera, F. Jaureguizar, and N. Garca, "MultiView Perceptual Disparity Model for Super MultiView Video," IEEE Journal of Selected Topics in Signal Processing, 2017.

[5] J. Wang, X. Xiao, H. Hua, and B. Javidi, "Augmented reality $3 \mathrm{D}$ displays with micro integral imaging," Journal of Display Technology, 2015.

[6] J.-Y. Son, "Viewing Zones of IP and Other Multi-view Image Methods," in Three-dimensional Imaging, Visualization, and Display, 2009.

[7] P. A. Kara, A. Cserkaszky, A. Barsi, T. Papp, M. G. Martini, and L. Bokor, "The Interdependence of Spatial and Angular Resolution in the Quality of Experience of Light Field Visualization," in International Conference on 3D Immersion (IC3D), 2017.

[8] D. Lanman and D. Luebke, "Near-eye light field displays," ACM Transactions on Graphics (TOG), 2013.

[9] A. J. Hansen, M. Kraus, and J. Klein, "Light field rendering for head mounted displays using pixel reprojection," in International Conference on Computer Graphics Theory and Applications, 2017.

[10] R. R. Tamboli, M. S. Reddy, P. A. Kara, M. G. Martini, S. S. Channappayya, and S. Jana, "A High-angularresolution Turntable Data-set for Experiments on Light 
Field Visualization Quality," in Tenth International Conference on Quality of Multimedia Experience (QoMEX), 2018.

[11] P. A. Kara, P. T. Kovács, M. G. Martini, A. Barsi, K. Lackner, and T. Balogh, "From a Different Point of View: How the Field of View of Light Field Displays affects the Willingness to Pay and to Use," in Eighth International Workshop on Quality of Multimedia Experience (QoMEX), 2016.

[12] R. R. Tamboli, B. Appina, S. Channappayya, and S. Jana, "Super-multiview content with high angular resolution: 3D quality assessment on horizontal-parallax lightfield display," Signal Processing: Image Communication, 2016.

[13] P. A. Kara, M. G. Martini, P. T. Kovács, S. Imre, A. Barsi, K. Lackner, and T. Balogh, "Perceived quality of angular resolution for light field displays and the validity of subjective assessment," in International Conference on 3D Imaging (IC3D), 2016.

[14] P. A. Kara, A. Cserkaszky, S. Darukumalli, A. Barsi, and M. G. Martini, "On the Edge of the Seat: Reduced Angular Resolution of a Light Field Cinema with Fixed Observer Positions," in 9th International Conference on Quality of Multimedia Experience (QoMEX), 2017.

[15] A. Cserkaszky, P. A. Kara, A. Barsi, and M. G. Martini, "To Interpolate or not to Interpolate: Subjective Assessment of Interpolation Performance on a Light Field Display," in IEEE International Conference on Multimedia and Expo (ICME) 8th Workshop on Hot Topics in $3 D$ Multimedia (Hot3D), 2017.

[16] —, "Expert Evaluation of a Novel Light-field Visualization Format," in 3DTV Conference, Stockholm, Sweden, 2018.

[17] S. Darukumalli, P. A. Kara, A. Barsi, M. G. Martini, T. Balogh, and A. Chehaibi, "Performance comparison of subjective assessment methodologies for light field displays," in International Symposium on Signal Processing and Information Technology (ISSPIT), 2016.

[18] P. T. Kovács, K. Lackner, A. Barsi, Á. Balázs, A. Boev, R. Bregović, and A. Gotchev, "Measurement of perceived spatial resolution in 3D light-field displays," in International Conference on Image Processing (ICIP), 2014.

[19] R. R. Tamboli, P. A. Kara, A. Cserkaszky, A. Barsi, M. G. Martini, and S. Jana, "Canonical 3D object orientation for interactive light-field visualization," in SPIE Applications of Digital Image Processing XLI, 2018.
[20] V. K. Adhikarla, P. Wozniak, A. Barsi, D. Singhal, P. T. Kovács, and T. Balogh, "Freehand interaction with large-scale 3D map data," in 3DTV-Conference: The True Vision-Capture, Transmission and Display of $3 D$ Video (3DTV-CON), 2014.

[21] V. K. Adhikarla, J. Sodnik, P. Szolgay, and G. Jakus, "Exploring direct 3D interaction for full horizontal parallax light field displays using leap motion controller," Sensors, 2015.

[22] A. Cserkaszky, P. A. Kara, R. R. Tamboli, A. Barsi, M. G. Martini, and T. Balogh, "Light-field capture and display systems: limitations, challenges, and potentials," in SPIE Novel Optical Systems Design and Optimization XXI, 2018.

[23] A. Cserkaszky, A. Barsi, Z. Nagy, G. Puhr, T. Balogh, and P. A. Kara, "Real-time light-field 3D telepresence," in 7th European Workshop on Visual Information Processing (EUVIP), 2018.

[24] V. K. Adhikarla, F. Marton, T. Balogh, and E. Gobbetti, "Real-time adaptive content retargeting for live multiview capture and light field display," The Visual Computer, 2015.

[25] A. Dricot, J. Jung, M. Cagnazzo, B. Pesquet, F. Dufaux, P. T. Kovács, and V. K. Adhikarla, "Subjective evaluation of super multi-view compressed contents on highend light-field 3D displays," Signal Processing: Image Communication, 2015.

[26] P. A. Kara, A. Cserkaszky, M. G. Martini, A. Barsi, L. Bokor, and T. Balogh, "Evaluation of the Concept of Dynamic Adaptive Streaming of Light Field Video," IEEE Transactions on Broadcasting, 2018.

[27] P. A. Kara, R. R. Tamboli, A. Cserkaszky, M. G. Martini, A. Barsi, and L. Bokor, "The perceived quality of light-field video services," in SPIE Applications of Digital Image Processing XLI, 2018.

[28] ITU-R Rec., "BT.500-13: Methodology for the subjective assessment of the quality of television pictures."

[29] P. T. Kovács, A. Fekete, K. Lackner, V. Adhikarla, A. Zare, and T. Balogh, "Big buck bunny light-field test sequences," in Proc. 112th Moving Picture Experts Group Meeting, 2015.

[30] ITU-T Rec., "P.910: Subjective video quality assessment methods for multimedia applications."

[31] P. A. Kara, Z. Nagy, M. G. Martini, and A. Barsi, "Cinema as large as life: Large-scale light field cinema system," in International Conference on 3D Immersion (IC3D), 2017. 